Clark, K. ; van Tongeren, M. ; Christensen, F.M. ; Brouwer, D. ; Nowack, B. ; Gottschalk, F. ; Micheletti, C. ; Schmid, K. ; Gerritsen, R. ; Aitken, R. ; Vaquero, C.; Gkanis, V. ; Housiadas, C.; Lopez de Ipina, J.M. ; Riediker, M.

Limitations and information needs for engineered nanomaterial-specific exposure estimation and scenarios: recommendations for improved reporting practices. Journal of Nanoparticle Research, 14 :970, 2012.

\begin{tabular}{|l|l|}
\hline Postprint version & Final draft post-refereeing \\
\hline Journal website & http://www.springerlink.com/content/103348/ \\
\hline DOI & $\underline{10.1007 / \mathrm{s} 11051-012-0970-\mathrm{x}}$ \\
\hline
\end{tabular}




\title{
Limitations and information needs for engineered nanomaterial- specific exposure estimation and scenarios: recommendations for improved reporting practices
}

\author{
Katherine Clark ${ }^{1}$, Martie van Tongeren ${ }^{2}$, Frans Christensen ${ }^{3 \$}$, Derk Brouwer ${ }^{4}$, Bernd \\ Nowack $^{5}$, Fadri Gottschalk ${ }^{5}$, Christian Micheletti ${ }^{4 \$}$, Kaspar Schmid ${ }^{2 *}$, Rianda Gerritsen ${ }^{3}$, Rob \\ Aitken $^{2}$, Celina Vaquero ${ }^{6}$, Vasileios Gkanis ${ }^{7}$, Christos Housiadas ${ }^{8,} J^{8}$ sús María López de \\ Ipiña $^{6}$, Michael Riediker $^{1 *}$
}

${ }^{1}$ Institute for Work and Health (IST), Lausanne, Switzerland KC-kclark@jhsph.edu, MR - Michael.riediker@hospvd.ch

${ }^{2}$ Institute of Occupational Medicine (IOM), Edinburgh, Scotland MvT-martie.vantongeren@iom-world.org; KS - kasparschmid@alumni.ethz.ch; RA: rob.aitken@iomworld.org

${ }^{3}$ Joint Research Centre (JRC), Institute for Health and Consumer Protection (IHCP), Ispra, Italy FC - frans.christensen@ec.europe.eu; CM - Christian.micheletti@gmail.com

${ }^{4}$ TNO, Zeist, The Netherlands DB- dick.brouwer@tno.nl; RG: rianda.gerritsen@tno.nl

${ }^{5}$ EMPA - Swiss Federal Laboratories for Materials Science and Technology, St. Gallen, Switzerland BN-nowack@empa.ch; FG - Fadri.Gottschalk@empa.ch

${ }^{6}$ TECNALIA Research and Innovation, Miñano, Spain CV-celina.vaquero@tecnalia.com; JL - jesus.lopezdeipina@tecnalia.com

${ }^{7}$ National Center for Scientific Research 'Demokritos', Athens, Greece VG - v_gkanis@hotmail.com; CH - christos@ipta.demokritos.gr

${ }^{\$}$ The opinions expressed in this paper are those of the authors and not necessarily those of the European Commission.

*currently affiliated with the Swiss Secretariat for Economic Affairs

Corresponding author:

Michael Riediker

Institute for Work and Health

Rue du Bugnon 21

1011 Lausanne

+41213147453(p)

+41213147430 (f)

michael.riediker@hospvd.ch 


\begin{abstract}
The aim of this paper is to describe the process and challenges in building exposure scenarios for engineered nanomaterials (ENM), using an exposure scenario format similar to that used for the European Chemicals regulation $(\mathrm{REACH})$. Over 60 exposure scenarios were developed based on information from publicly-available sources (literature, books, and reports), publicly-available exposure estimation models, occupational sampling campaign data from partnering institutions, and industrial partners regarding their own facilities. The primary focus was on carbon-based nanomaterials, nano-silver (nano-Ag) and nano-titanium dioxide (nano- $\mathrm{TiO}_{2}$ ), and included occupational and consumer uses of these materials with consideration of the associated environmental release. The process of building exposure scenarios illustrated the availability and limitations of existing information and exposure assessment tools for characterizing exposure to ENM, particularly as it relates to risk assessment. This article describes the gaps in the information reviewed, recommends future areas of ENM exposure research, and proposes types of information that should, at a minimum, be included when reporting the results of such research, so that the information is useful in a wider context.
\end{abstract}

\title{
Keywords
}

Nanomaterials, exposure assessment, risk assessment, modeling, REACH 


\section{Introduction}

Materials at the nano-scale often have differing or unique properties, compared to their larger counterparts, such as improved electrical conductivity or biocidal activity. Growth in nanotechnology, coupled with enthusiasm for its potential market penetration, has raised concerns among health and environmental scientists about the potential risks of engineered nanomaterials (ENMs) to human health and the environment (Balbus et al. 2007; ICON 2008; Royal Society 2004). The hazards of exposure to some types of particles and dust (e.g., coal dust, asbestos, silica) have been known for centuries and there is strong epidemiologic evidence of the link between exposure to ambient particulate and cardiovascular morbidity and mortality (US EPA 2009). ENMs may be particularly potent, as their small size and corresponding large surface area to mass ratio may lead to increased ability to induce oxidative stress, more efficient penetration of biological barriers, and undesirable interaction with biological macromolecules (Donaldson et al. 2004; Maynard et al. 2011; Nyland and Silbergeld 2009; Oberdorster et al. 2005). Similarly, their small size and surface properties may result in higher mobility, uptake, bioaccumulation and/or environmental toxicity following environmental release.

There has been a significant increase in research on nanomaterial risk assessment and management, as analyses on the increasing annual number of publications in this area demonstrate (Linkov et al. 2009; Maynard et al. 2011). Despite this increase, there is significant uncertainty concerning the risks posed by ENMs (Savolainen et al. 2010). The challenges to assessing the risks of ENMs stem from their chemical and functional diversity, limited methodology to detect and quantify these materials in complex environments, and uncertainty regarding the degree to which they are or will be used in industry or consumer applications (Bouwmeester et al. 2011).

\section{Exposure scenarios}

Exposure assessment is a critical step in the risk assessment process. Exposure has been defined as the 'contact between an agent and a target', while exposure assessment is 'the process of estimating or measuring the magnitude, frequency and duration of exposure to an agent, along with the number and characteristics of the population exposed' (Zartarian et al. 2005). Detailed knowledge of the conditions under which exposure occurs are a critical part of the exposure assessment process, as factors such as frequency and duration of exposure, presence of certain activities or processes, and the use of risk management measures can significantly mitigate or intensify exposure (and thus risk). In recognition of this, the new European Chemicals regulation REACH has introduced the concept of Exposure Scenarios as the operational conditions and risk management measures based on which exposure is estimated. To support implementation of REACH, the European Chemicals Agency (ECHA) has issued guidance, including a format for describing occupational and consumer exposure scenarios, both of which include consideration of environmental release. In addition to the operational conditions and risk management measures, the format also contains the exposure estimates associated with the ES (ECHA 2008).

This paper describes the conclusions and recommendations of a multi-institutional project funded by the European Commission Framework Program 7 with the stated goal of developing a catalogue of exposure scenarios and associated exposure estimates for engineered nanomaterials (ENM), taking account of the entire lifecycle of these materials (van Tongeren et al, 2011). Exposure scenarios and estimates were recorded in the format outlined by ECHA. However, there is an important distinction between the exposure scenarios developed 
in this project and those developed specifically for the purposes of meeting REACH requirements. A REACH exposure scenario not only provides the basis for estimating occupational, consumer, and environmental exposure: it is also the output of the iterative chemical safety assessment process and should set out the conditions for safe use of a substance. The exposure scenarios discussed in this article outline the conditions of use and associated exposure estimations based on available data. They are, however, not validated against noeffect-levels as it was outside the scope to derive such thresholds. Thus, it must be emphasized that the term 'Exposure Scenario' as used in this article does not refer to any 'final' Exposure Scenario, in REACH terminology, which has been shown to control risks.

In developing exposure scenarios, efforts were focused primarily on exposure scenarios for carbon nanotubes (CNTs), nano-silver (nano-Ag) and nano-titanium dioxide (nano- $\mathrm{TiO}_{2}$ ), although additional nanomaterials were included in some cases if they were thought to demonstrate similar properties (e.g., carbon nanofibers or other metal oxides). Over 60 exposure scenarios were developed related to production and products containing ENM and professional and consumer uses of such products. The information used to develop the exposure scenarios and associated exposure estimates included publicly-available information, publicly-available exposure estimation models, occupational sampling campaign data from partnering institutions, and information from industrial partners regarding their own facilities. The process of building exposure scenarios illustrated the availability and limitations of information and exposure assessment tools for characterizing exposure to ENM, particularly as it relates to risk assessment.

This article integrates the findings and conclusions of experts in areas of occupational exposure, consumer exposure, and environmental release and presents recommendations for future research including concrete proposals for a minimum set of information, which should be included when reporting results. More detailed discussion of the project conclusions can be found in associated peer-reviewed journal articles and project reports (Gottschalk and Nowack 2011; van Tongeren et al 2011).

\section{Process}

The key tasks in this project included conducting a literature review, evaluating tools for exposure assessment, and developing exposure scenarios (Figure 1). For each step in the process the information was evaluated for completeness, relevance, and/or utility relative to exposure scenarios.

The literature review was intended to survey the existing publicly available information on nanomaterials. The specific articles included in the literature review were selected by a systematic literature search. (All articles reviewed are referenced in the supplementary information). Information from sources which were found to contain information relevant for building ESs was stored in two libraries that were specifically developed for this project. The first, the Reference library, documented the data and characteristics of the references. The second, the Exposure Scenario library, stored the developed exposure scenarios in a format based on that used for REACH compliance.

The references were not evaluated against a strict weighting or quality assessment criteria. Such an evaluation was considered, but as there are no broadly accepted guidelines delineating the key elements of exposure 
assessment for ENM (e.g., exposure metrics and analytical methods), this was not considered feasible or defensible. Instead, a detailed list of points that were broadly considered potentially important for nanomaterial exposure assessment was evaluated in each study (Table 1). Details that were missing from studies (such as task activity, duration, or number of samples collected) were noted and the study reviewers subjectively evaluated methodological quality of each study and relevance to building exposure scenarios.

Exposure scenarios were developed using the information from the literature survey, data from the NANOSH and Nano-Innov sampling campaigns, or from information provided by industry partners (http://www.ttl.fi/partner/nanosh/Sivut/default.aspx and .(www.cea.fr). In some cases, output from exposure estimation models, such as ECETOC-TRA (ECETOC 20110 and ConsExpo (www.rivm.nl), were used to supplement exposure scenarios. The NANOSH project (funded by the Framework Programme 6 of the European Commission) conducted air sampling at several worksites throughout Europe. The Nano-INNOV project, run by CEA (Comissariat à l'Energie Atomique et aux Energies Alternatives) in France, collected samples at worksites and laboratories handling or generating ENMs throughout France. Once the development of the exposure scenarios was complete, the libraries containing the exposure scenarios and literature information were analyzed for completeness, breadth, and overall level of confidence in the exposure estimates. A quantitative analysis of the library is inherently limited by the nature of the information collected, the differences in opinion of the scientists reviewing the studies, and the small number of studies that exist on ENM exposure (in particular for consumer exposure), but it allowed for the assessment of the strengths and weaknesses of currently available exposure data for building exposure scenarios.

The following exposure scenarios and the associated exposure estimates were developed/described, according to source of information:

- 22 occupational exposure scenarios based on publicly available literature.

- 35 occupational exposure scenarios based on sampling campaign data from the NANOSH and NanoINNOV projects.

- 5 consumer exposure scenarios based on publicly available literature and models.

- 12 exposure scenarios based on industry case illustrations (for confidentiality reasons, these were not included in the Exposure Scenario library).

It was not possible, due to limitations in available information and data, to develop contributing environmental exposure scenarios for any of the above mentioned scenarios, with the exception of those based on industry case illustrations.

The process of translating scientific measurements into the exposure scenario format revealed that there are many challenges to developing well-supported exposure scenarios for ENM using the existing information. Many of the exposure scenarios were either missing significant amounts of information needed to characterize the operational conditions, risk management measures and/or the exposure, or were developed using information with questionable relevance or reliability. Although the original intent of the project was to create a publicly available database of nanomaterial exposure scenarios, only a small portion of the exposure scenarios that were 
developed have been made publicly available to avoid misinterpretation (van Tongeren et al 2011).

The following sections discuss in more detail the areas in which current exposure assessment practices which were found to be deficient for the purposes of building exposure scenarios. The details of the exposure scenarios themselves and more quantitative analyses of the scenarios and literature are not provided here, but are available from project reports (http://www.nanex-project.eu) and forthcoming peer-reviewed articles. The discussion is followed by concrete proposals for future research needs and a minimum set of data, which could improve the current data deficiencies.

\section{Limitations in exposure data}

Quantity and scope of available information

Fewer than 30 publicly available references (of the over 60 reviewed) were considered to contain data that could be used to describe exposure scenarios, including exposure estimates for ENM of interest. The remainder either lacked key information needed for evaluating exposure to ENMs, such as information on risk management measures, results of exposure measurements and adequate characteristics of material under study, or were only marginally relevant to the situations of interest (e.g., medical uses of certain ENMs). The distribution of situations described by these articles was weighted towards occupational exposure during pilot/ small-scale manufacture or laboratory use of ENM, with less available on downstream uses of ENM (e.g., creation or use of products containing ENM) in occupational or consumer settings. The data from sampling campaigns were generally more detailed and covered a wider range of activities, but were mostly focused on research and development activities and did not always include detailed descriptions of the material under study, measures to prevent environmental release, or use patterns of the ENMs.

The lack of quantitative consumer exposure data for ENMs as well as contextual exposure information severely limited the ability to develop exposure scenarios for consumer uses of products containing ENM. A small number of hypothetical consumer exposure scenarios were developed: for lack of quantitative data, exposure estimates associated with all consumer exposure scenarios were obtained using exposure estimation models (e.g., ECETOC-TRA and ConsExpo), recognizing that these models have neither been calibrated nor validated for ENM.

One of the most significant gaps observed was the limited amount of identified information that described exposure over the ENM life cycle, including occupational exposure during full-scale and down-stream uses, consumer and occupational exposure during use of products containing ENM, or environmental release.

\section{Interpretation and utility of existing human exposure information}

Exposure to chemical agents can generally be defined by a single metric (e.g., mass per volume). However, for ENM it is generally agreed that there is no single exposure metric that is able to sufficiently characterize exposure to ENM for the purposes of ENM exposure and risk assessment (Bouwmeester et al. 2011). ENM exposure studies commonly measure particle number size distribution, particle number concentration, and/or mass concentration, but there is no consistency in choice of measurements between studies. In addition, it is not 
clear which of these, if any or if in combination, are most relevant for human health. Evidence also suggests that measurement of surface area is very relevant (Borm et al. 2006).

In the occupational setting, exposure to airborne substances is typically evaluated in the worker's breathing zone (Nieuwenhuijsen 2003). The studies reviewed tended to measure emission from a source, with limited descriptions of the conditions which may affect worker exposure (e.g., distance from source, presence of ventilation, frequency and duration of activity). In order to quantify and characterize occupational exposure (and associated risks), personal measurements of exposure should ideally be carried out. If this is not possible, then the relationship between the source, receptor, and conditions of exposure must be well described.

The characteristics that are most important in determining exposure to ENMs, which may be associated with particle characteristics or with the exposure setting, still need to be identified (Brouwer 2010). The level of detail reported in the literature is often not sufficient to allow for detailed understanding of the situation under study, comparisons of results to those of other studies, or for future re-analysis of the data. It was common for studies to not fully describe how many samples were collected, how results were averaged, if and when there were peaks in concentration, how background was measured, or how final measurements were adjusted relative to background. In terms of process characteristics, many studies did not elaborate on the characteristics of ENM under study (beyond the name of the ENM), amounts used, frequency and duration of activities, or risk management measures in place during measurements. Small changes in these factors can affect the propensity for ENM to be released and their behavior and fate following release.

\section{Methodological and technical challenges for estimation of human exposure and environmental release}

Qualitative and quantitative identification of ENM is complex, as these materials have very low mass, can be highly dynamic in terms of particle aggregation or reactivity, co-exist with ambient particles in the same size range, and also often co-exist with molecules or macro-sized counterparts of the same chemical composition. The instrumentation involved in detecting and identifying ENMs is expensive and requires specialized training, both for instrument operation and interpretation of results. Many of the instruments used in such analyses are heavy and more easily used in stationary settings and not suitable to collect human personal exposure measurements. Often a tiered approach is used, where for the various tiers the complexity of the devices will increase.

Studies of inhalation exposure to ENMs tend to use a combination of particle counters and electron microscopy: the former counts the number concentration of all particles within a certain size range (dictated by the instrument), and the latter is used to further characterize and identify the particles counted (Methner et al. 2010; Methner et al. 2007). Since the particle counters are not specific for ENMs, background distinction in these measurements is an important issue (Brouwer 2010). Transmission electron microscopy is a useful analytical tool for confirming the presence of ENM in an aerosol sample, but has limitations for quantification related to uncertain efficiency and homogeneity of deposition, is labor intensive and requires potentially disruptive sample preparation. 
The treatment of background aerosols when measuring for ENMs presents a particularly challenging problem. Several approaches have been proposed and applied, including subtraction of background concentrations, either measured prior to the activity or during the activity away from the source, and statistical techniques. However, concurrent processes (i.e., use of combustion or electro motors) can be a significant source of other (potentially health relevant) particles (Koponen et al. 2010; Szymczak et al. 2007). Furthermore, these techniques for correcting background levels do not account or the interactions between ambient aerosols and the ENM particles.

When aerosolized, ENM coagulate homogeneously, or heterogeneously with other ENM, or attach to ambient background particles; all processes effectively alter the particle size distribution, the particle number concentration, and the chemical composition of the background aerosols (Schneider et al. 2011; Seipenbusch et al. 2008). Many studies of aerosolized ENM focus primarily on measuring nano-sized particles (compared to larger particles), yet particles may no longer be in the nano-size range at the time of sampling.

In contrast to occupational and consumer exposure assessments, environmental release of ENM has to consider a variety of diffusive emission sources that cover the whole life cycle of ENM and ENM-containing products (Gottschalk and Nowack 2011). This life cycle perspective has to distinguish between release from ENM production, ENM incorporation into products, product use and recycling or disposal of the ENM-containing products. It is therefore crucial to assess parameters such as ENM production, application and use in order to frame ENM emission models as well as experimental and analytic studies. Unintentional release during use is the most important release scenario for ENM. Several studies have provided evidence that ENM are unintentionally released from different products, e.g. release of silver from textiles during washing (Geranio et al. 2009) or release of nano- $\mathrm{TiO}_{2}$ from paints due to weathering (Kaegi et al. 2008). In other applications the release of ENM during use is practically inevitable, such as when ENM are used in sunscreens or cosmetics (Auffan et al. 2010).

It is essential to know if the ENM are strongly embedded within a solid material matrix, present in liquid form in emulsions or suspensions or even used as aerosols that are emitted directly to the air. A significant release of ENM is expected from use of liquids, pastes, creams, powders or aerosol sprays containing ENMs. Also ENM coated onto surfaces may be emitted in significant amounts during use of the product (e.g., a textile). Mechanical abrasion and physicochemical aging of materials may impact considerably the release of ENM (Köhler et al. 2008). It is extremely difficult to quantify and monitor the release of ENM from products and to characterize the released materials. Analytical challenges are to identify a few ENM within a large background of natural nanoparticles or larger particles (von der Kammer et al. 2011).

\section{Life cycle approach to evaluating exposure}

Characterizing the life cycle of a material/product is important for understanding at which life stage(s) a material may pose risks to humans or the environment, and for subsequent risk management. Although the intent of this project was to consider the lifecycle of ENM, due to the lack of relevant information available this was not feasible. Many of the studies that were reviewed focused on occupational or, to a lesser extent, consumer exposure at one specific stage of the life cycle of ENM or ENM-containing products. There are many missed opportunities for collecting information relevant to ENM life cycle. For example, site-specific studies could 
report volumes of ENM used or information on disposal/recycling practices of ENM-containing products (assuming that the industrial site was willing to share that information). Such information will allow for the link to be made between sector-specific use of ENMs and their release and concentration in the environment.

\section{Use of exposure estimation models for ENM}

It is not feasible to take measurements for every possible exposure scenario, especially given the technical difficulties in measuring ENM. Thus, available exposure estimation models that are calibrated and validated to ENMs, for both human exposure and environmental release, are urgently needed. The development of such models is contingent upon the collection of well-documented and representative ENM exposure and release measurements.

\section{Environmental release}

When estimating environmental release, one must consider, besides local/point emissions, an immense diffusive emission source covering the whole life cycle of ENM and ENM-containing products. The data currently available cover only a small fraction of that which is required in such a release framework. There is limited scientific information available on ENM environmental release or fate in the environment and studies on occupational and environmental exposure typically do not contain information relevant for estimating environmental release. Mathematical modeling is currently the most powerful tool available for estimating environmental release, yet the models that exist are characterized by high parameter uncertainty.

The first equations to predict emissions of ENMs into air, soil, and water were based on hypothetical usage scenarios of single product-types and life cycle stages of ENM (Boxall et al. 2008). Subsequent mass balance multi-compartment models (Gottschalk et al. 2009) suggested that the most commonly used metallic ENM (i.e., nano-Ag, nano- $\mathrm{TiO}_{2}$, nano-ZnO) are transferred from products to sewage treatment plants (STP), sewage treatment plants to waste incineration processes (WIP), and from there to landfills. For CNTs, the majority of the emitted material is likely to end up in waste incineration plants. Results from a comprehensive sensitivity analysis identified the key parameters that govern ENM environmental release and exposure (Gottschalk et al. 2010). These include the release of ENM from products to the sewage treatment plant (STP), STP removal efficiency, and the overflow and sludge from this compartment which can end up in the waste incineration plant or deposited in landfills. The most dominant parameter - however, associated with high uncertainties - is the assignment of the mass of ENM that could be released from the products that are seen as main ENM emitters in such modeling. Quantitative data on ENM emissions (in terms of mass release rate) to the various environment compartments during the manufacturing of ENM-containing products and during the production of ENM is urgently needed.

Models for human exposure

Two risk banding tools are currently available for ENMs, which include a module to estimate exposure to ENMs. Stoffenmanager Nano uses a qualitative exposure estimation module (www.stoffenmanager.nl), while NanoSafer uses a quantitative exposure module (http://nanosafer.i-bar.dk). Both modules are based on the 
concepts of inhalation exposure modeling for nanoparticles as proposed by Schneider et al, 2011. Unfortunately, these have only become available following the completion of our study. Instead, existing publicly available generic quantitative exposure estimation models for conventional substances were reviewed to determine relevance to estimating ENM exposure in various situations. The models reviewed included Stoffenmanager and ECETOC TRA for estimating occupational inhalation exposure and RiskofDerm (ROD), ConsExpo, ECETOC TRA, and the Spray Paint model for estimating consumer exposure (dermal and/or inhalation) (Brouwer et al. 2001; ECETOC 2011; Marquart et al. 2008; van Hemmen et al. 2003; van Veen 1995). For each, the theoretical basis of the model was evaluated to determine if modifications to the model parameters and / or equations are required when used to estimate exposure to ENMs.

The ConsExpo evaporation model and the Spray Paint model were not considered applicable to ENM, due to inconsistencies between the scope of the model and the properties or uses of ENM. For dermal models, nanospecific properties are not expected to be critical in determining exposure (i.e., amount of particles coming into contact with skin; these models do not address dermal uptake). However, it is not clear if the use patterns for nano-enabled products are different than for other products. Further, the output of these models is given in the mass metric only, which may not be the best way to describe exposure to ENM. Given these qualifiers, the dermal models could only be used to estimate ENM exposure with appropriate caution.

For inhalation models, the current models are not applicable or at best probably not very accurate for ENM. As with the dermal models, all of the inhalation models provide estimates in terms of mass concentration. It is not known in what quantities and forms ENM are released during various activities, and whether this is different than for activities with more traditional substances. Further, the existing models do not account for nano-specific factors that affect ENM behavior in air, such as agglomeration of particles and the influence of background aerosols on agglomeration rates or particle size (Schneider et al. 2011). One particular limitation of applying the ECETOC TRA model to estimate occupational exposures to ENM is that it uses activities that are defined in terms of process categories (PROCs) as the basis for crude Tier 1 estimates of exposure. PROCs are also used to describe activities in the REACH exposure scenario format and tend to describe general processes (e.g., "Use in closed batch process [synthesis or formulation]") (ECHA 2008). As most studies on occupational exposure to ENM involve short term samples during specific activities, it is difficult to calibrate, validate, or compare the modeled estimates to experimental data.

To our knowledge, these generic exposure estimation models have not been evaluated for ENMS using actual exposure measurement data. Using data from the NANOSH and NanoINNOV sampling campaigns, occupational exposure (mass) concentrations for a range of PROCs predicted by the models were compared to the particle concentrations measured in the field. Results showed that the model estimates were not correlated (rank test) with the measurement results of actual concentrations of nanoparticles in workplaces.

\section{Evaluation of the case study exposure scenarios}

Exposure scenarios were developed with industrial partners covering their own activities involving ENM. The purpose of the case studies was to compare the process of developing exposures scenarios based on 
collaborations with industrial partners and the quality of the available data with that of exposure scenarios developed based on existing information from the survey and literature. Although the case study exposure scenarios were far more detailed in terms of process and material characteristics than those based on sampling campaigns or the literature, there were similar difficulties in terms of quantifying and characterizing the exposure. The case study providers did not always have measurement data from their sites, and in a number of cases had to rely on exposure estimation models. None of the exposure data from the peer-reviewed literature were deemed to be suitable for providing exposure estimates, by the process of 'read across'. Although the case study providers could provide more detailed descriptions of potential for release of ENMs to the environment and control measures to limit the release and exposure, quantitative data on amounts of ENMs released from their facilities were generally not available. Additionally, the case study provides could not provide detailed information available on potential for exposure from downstream use of professional or consumer uses of their products. These difficulties underscore the need for better tools for nanomaterial exposure assessment.

\section{Research priorities}

We have identified several specific ENM research needs for building an improved library of exposure scenarios. It was clear in our view that a coherent strategy for exposure assessment, as it relates to risk assessment, needs further development. Table 2 provides a list of research priorities, as well as a timeline for accomplishing these research needs. In the short term, two lines of research should be established; one focusing on establishing the effectiveness of risk management strategies for ENMs and the other focusing on the basic needs for more detailed and consistent research. The latter line of research should lead to a better understanding of the relationship between operational conditions and exposure, which would lead to development of more accurate and reliable exposure models. In the medium to long term, with additional and more harmonized research into exposure (and hazards) of ENM, more detailed risk assessments should become feasible.

\section{Recommendations for a minimum set of data items for reporting of exposure studies on engineered nanomaterials}

Several limitations in technology and interpretation of measurement data were identified. Some of these limitations will take considerable time to address, particularly technical methods for measuring relevant exposure metrics and establishment of exposure-response relationships. One area where immediate improvement is possible is in more harmonized or standardized reporting of exposure studies, including sampling strategy, collection and description of contextual information (e.g., operational conditions and risk management measures), and data handling. It was this information that was often not reported, limiting the possibility to extrapolate the data to other situations (i.e., for building exposure scenarios)

Contextual information is essential for interpretation of the exposure measurement results and exposure assessment (e.g., when translating results from stationary measurements into exposure estimates). As noted previously, basic information about ENM use and release volumes (and rates) from a site could contribute significantly to the limited existing body of information on environmental release. Further, contextual information is necessary for identifying how factors such as operational conditions or risk management measures 
can affect the magnitude of exposure and aerosol properties (e.g., agglomeration and transmission). Ideally, it will be possible to estimate the impact of operational conditions and risk management measures on measurement results and exposure estimates. This level of understanding of ENM exposure is needed to build models, compare studies, understand the relevance of information collected in one situation for another situation (readacross), and, thus, to build exposure scenarios. We propose a minimum set of items that should be reported for all ENM exposure studies, which includes both nano-specific and non-nano-specific items.

Nano-specific information:

- Description of physical and chemical form of the ENM used (i.e., at source);

- Chemical composition, including surface treatment/modification

- $\quad$ Size distribution (including dimensions for fibres)

- Surface area

- Details on the matrix surrounding the ENM (if any):

The matrix itself (e.g., plastic, rubber, concrete, paint)

Form of matrix (e.g., powder, liquid, solid, granules)

Amount of ENM used in the matrix

- Description of physical and chemical form of released/detected particles;

- $\quad$ Embedded in a matrix, agglomerated, single particle

- Elemental composition by EDX/EDS or chemical analysis

- Potential other sources of ultrafine and other particles; and

- Human exposure characterized using a combination of metrics and measurements, which could include, but are not limited to, mass, particle number, surface area, and particle size distribution.

- Environmental release characterized in terms of mass flow rates and not only concentrations

Information that is not nano-specific:

- Information on process

- Description of the process and all activities included in the process;

- $\quad$ Typical duration and frequency of these activities; and

- Type of enclosure of process: if enclosed, provide frequency and duration of opening for maintenance, quality control and/or other manual operations.

- Description of site

- $\quad$ Room size, windows and other features that may affect exposure.

- $\quad$ Risk management measures (RMM)

- $\quad$ For occupational studies, standardized description of types of RMM (e.g. ventilation) and personal protective equipment in use (Fransman et al. 2009);

- For consumer studies, product design that affects the release (e.g., maximum volume released from one use of a spray) and description of other types of RMM applied during the measurement; and

- Other measures to prevent human exposure or environmental release (e.g., administrative controls, additional engineering controls).

- Environmental release information 
- Total volume of ENM used on site;

- Amounts and processes for disposal and/or recycling;

- Volume of air flow and ENM concentrations in outlet air (emission to air after filters); and

- Volume of wastewater flow and ENM concentrations in effluent (after treatment) (emission to surface waters).

- Sampling and data analysis strategy for human exposure:

- $\quad$ Location of samples/measurements relative to source and receptor (e.g., workers);

- Number of samples/measurements taken;

- Description of activities associated with each sample/measurement;

- Qualitative assessment describing how representative the measurements are for personal exposure;

- Description of data analysis, including the difference between background and activity and how this was calculated, whether and how peaks were addressed, whether and how data were averaged.

This list represents the minimum data that we believe exposure scientists should report when describing the results of an exposure assessment study. We encourage scientists to also include the following information:

- Description of activities or use of RMMs in a standardized format, such as that in the Advanced REACH Tool (Fransman et al. 2009).

- More detailed description of ENM physicochemical properties, including surface reactivity, and solubility/biopersistence.

It is dependent upon the expert judgment of the exposure assessor to include additional information when possible or deemed necessary (e.g., additional sources of environmental release or exceptions to standard operating procedures on the day of the study).

The minimum dataset does not include a prescribed list of measurements needed in an exposure assessment: this is because there is still significant uncertainty on this issue, and it was not within the scope of this project to determine the best choice of metrics or measurements. It is clear, however, that there is currently no generally accepted single metric that can sufficiently characterize and quantify ENM exposure for all types of ENM and that exposure is best characterized by a combination of metrics. In the interim, until there are well-supported recommendations for presenting such data, we suggest that exposure studies include as much information as possible in terms of measurements, recognizing that not all may be appropriate in every situations. Size distribution is important for understanding the likelihood of deposition of particles in certain parts of the airways, while surface area concentration is probably more associated with potential toxicity of an ENM. The mass concentration is important because there is already a large body of research on exposure to and toxicity of particles in the mass-based metric, making these data more comparable. Finally, particle (or fiber) number concentration is important as this metric may, in some cases, be more relevant than mass in determining potential risk from exposure to ENM and because the mass of airborne nanoparticles will usually be very small, making it more difficult to measure than particle number. 
The importance of harmonizing the metrics/measurements used to describe exposure, so that data can be compared, pooled, and used for broader research (e.g., epidemiology studies and development of nano-specific models) cannot be overstated. Although strict harmonization can inhibit innovation in research, a certain level of congruency is needed. It is imperative that harmonized basic guidelines, preferably internationally agreed upon, for measurements collection and reporting be developed in the near future. Such initiatives for international collaboration in the area of occupational exposure have been taken (Brouwer et al.2011).

Much of the contextual information requested above is easily observed and assembled. This level of detail can become burdensome to publish in peer reviewed journal articles due to space limitations; if so, we recommend making this information available in appendices or online supplementary information for articles and reports.

\section{Conclusions}

An exposure scenario, whether developed for REACH compliance or otherwise, can be a powerful tool for understanding the conditions under which exposure occurs (i.e., amounts used, operational conditions and risk management measures), as long as operational conditions and risk management measurements are comprehensively described and exposure has been assessed using high quality exposure measurements methods. This information can be used to identify both safe work practices and those that could be considered unacceptable risky. The ability to build comprehensive exposure scenarios and estimate associated exposure for ENM is fundamental to risk evaluation, both at the site-specific level and at a more global level. For ENMs, the skills and instrumentation needed to assess exposure can be costly, limiting the ability of many companies and organizations to characterize the range of exposure scenarios associated with their processes and products.

Publication and dissemination of comprehensive exposure scenario descriptions could contribute to promotion of better product stewardship.

Predictably, one of the key messages of this paper is that significantly more research is needed before comprehensive exposure scenarios and associated exposure estimates ENM can be developed for ENM in the range of products expected to contain ENMs. However, a perhaps more important message is that there are several missed opportunities in existing research to provide detailed descriptions of production processes, product uses, and sampling strategies. Although such details may not have immediate bearing on the hypotheses that motivate individual studies, they could make the difference on whether the data in question is useful for inclusion in larger pooled datasets (e.g., for assessing the efficacy of risk management measures, understanding aerosol behavior, building models, or making exposure registries). The field of ENM exposure assessment is still in its infancy: researchers in this field can contribute to advancement of the field and maximize the impact of their own research by better describing and sharing the details of their research.

\section{Acknowledgments}

This study was supported by the European Commission's 7th Framework Programme Coordination and Support Action "NANEX - Development of Exposure Scenarios for Manufactured Nanomaterials" (Grant NMP-CSA247794). 
We thank the Dr. Steffi Friedrichs, the Nanotechnology Industries Association (NIA), and its members for their generous cooperation and assistance with the case studies. 


\section{References}

Auffan M, Pedeutour M, Rose J, Masion A, Ziarelli F, Borschneck D, Chaneac C, Botta C, Chaurand P, Labille J, Bottero JY (2010) Structural degradation at the surface of a $\mathrm{TiO}_{2}$-based nanomaterial used in cosmetics. Environ Sci Technol 44: 2689-2694

Balbus JM, Maynard AD, Colvin VL, Castranova V, Daston GP, Denison RA, Dreher KL, Goering PL, Goldberg AM, Kulinowski KM, Monteiro-Riviere NA, Oberdorster G, Omenn GS, Pinkerton KE, Ramos KS, Rest KM, Sass JB, Silbergeld EK, Wong BA (2007) Meeting report: Hazard assessment for nanoparticles--report from an interdisciplinary workshop. Environ Health Perspect 115: 1654-1659

Borm PJ, Robbins D, Haubold S, Kuhlbusch T, Fissan H, Donaldson K, Schins R, Stone V, Kreyling W, Lademann J, Krutmann J, Warheit D, Oberdorster E (2006) The potential risks of nanomaterials: A review carried out for ecetoc. Part Fibre Toxicol 3: 11

Bouwmeester H, Lynch I, Marvin HJ, Dawson KA, Berges M, Braguer D, Byrne HJ, Casey A, Chambers G, Clift MJ, Elia G, Fernandes TF, Fjellsbo LB, Hatto P, Juillerat L, Klein C, Kreyling WG, Nickel C, Riediker M, Stone V (2011) Minimal analytical characterization of engineered nanomaterials needed for hazard assessment in biological matrices. Nanotoxicology 5: 1-11

Boxall ABA, Chaudhry Q, Jones A, Jefferson B, Watts CD (2008) Current and future predicted environmental exposure to engineered nanoparticles. Central Science Laboratory. Sand Hutton, UK.

Brouwer D (2010) Exposure to manufactured nanoparticles in different workplaces. Toxicology 269: 120-127

Brouwer DH, Berges M, Virji MA, Fransman W, Bello D, Hodson L, Gabriel S, Tielemans E Harmonization of measurement strategies for exposure to manufactured nano-objects: Report of a workshop. Annal Occup Hyg doi: 10.1093/annhyg/mer099

Brouwer DH, Semple S, Marquart J, Cherrie JW (2001) A dermal model for spray painters. Part i: Subjective exposure modelling of spray paint deposition. Ann Occup Hyg 45: 15-23

Donaldson K, Stone V, Tran CL, Kreyling W, Borm PJ (2004) Nanotoxicology. Occup Environ Med 61: 727 728

ECETOC. (2011). "Targeted risk assessment (tra) tools." Retrieved May 25, 2011, from http://www.ecetoc.org/tra.

ECHA (2008) Guidance on information requirements and chemical safety assessment. Part d: Exposure scenario building. European Chemicals Agency (ECHA). Helsinki, Finland. Version 1.1.

Fransman W, Cherrie J, van Tongeren M, Schneider T, Tischer M, Schinkel J, Marquart H, Warren N, Kromhout $\mathrm{H}$, Tielemans E (2009) Development of a mechanistic model for the advanced reach tool (art). TNO Quality of Life. Zeist, The Netherlands.

Geranio L, Heuberger M, Nowack B (2009) The behavior of silver nanotextiles during washing. Environ Sci Technol 43: 8113-8118

Gottschalk F, Nowack B (2011) The release of engineered nanomaterials to the environment. J Environ Monit 13: 1145-1155

Gottschalk F, Sonderer T, Scholz RW, Nowack B (2009) Modeled environmental concentrations of engineered nanomaterials $\left(\mathrm{TiO}_{2}, \mathrm{ZnO}, \mathrm{Ag}, \mathrm{CNT}\right.$, fullerenes) for different regions. Environ Sci Technol 43: 9216 9222

Gottschalk F, Sonderer T, Scholz RW, Nowack B (2010) Possibilities and limitations of modeling environmental exposure to engineered nanomaterials by probabilistic material flow analysis. Environ Toxicol Chem 29: $1036-1048$

ICON (2008) Towards predicting nano-biointeractions: An international assessment of nanotechnology environment, health and safety research needs. International Council on Nanotechnology; Rice 
University. Houston, TX.

Kaegi R, Ulrich A, Sinnet B, Vonbank R, Wichser A, Zuleeg S, Simmler H, Brunner S, Vonmont H, Burkhardt $\mathrm{M}$, Boller M (2008) Synthetic $\mathrm{TiO}_{2}$ nanoparticle emission from exterior facades into the aquatic environment. Environ Pollut 156: 233-239

Köhler A, Som C, Helland A, Gottschalk F (2008) Studying the potential release of carbon nanotubes throughout the application life cycle. Journal of Cleaner Production 16: 927-937

Koponen IK, Jensen KA, Schneider T (2011) Comparison of dust released from sanding conventional and nanoparticle-doped wall and wood coatings. J Expo Sci Environ Epidemiol 21: 408-415

Linkov I, Satterstrom FK, Monica JCJ, Hansen SF, Davis TA (2009) Nano risk governance: Current developments and future perspectives. Technology Law \& Business 202: 203-220

Marquart H, Heussen H, Le Feber M, Noy D, Tielemans E, Schinkel J, West J, Van Der Schaaf D (2008) 'Stoffenmanager', a web-based control banding tool using an exposure process model. Ann Occup Hyg 52: 429-441

Maynard AD, Warheit DB, Philbert MA (2011) The new toxicology of sophisticated materials: Nanotoxicology and beyond. Toxicol Sci 120 Suppl 1: S109-129

Methner M, Hodson L, Geraci C (2010) Nanoparticle emission assessment technique (neat) for the identification and measurement of potential inhalation exposure to engineered nanomaterials--part a. J Occup Environ Hyg 7: 127-132

Methner MM, Birch ME, Evans DE, Ku BK, Crouch K, Hoover MD (2007) Identification and characterization of potential sources of worker exposure to carbon nanofibers during polymer composite laboratory operations. J Occup Environ Hyg 4: D125-130

Nieuwenhuijsen M, Ed. (2003). Exposure assessment in occupational and environmental epidemiology, Oxford University Press.

NNI (2011) National nanotechnology initiative strategic plan. National Nanotechnology Initiative; National Science and Technology Council; Committee on Technology; Subcommittee on Nanoscale Science, Engineering, and Technology. Washington DC.

Nyland JF, Silbergeld EK (2009) A nanobiological approach to nanotoxicology. Hum Exp Toxicol 28: 393-400

Oberdorster G, Oberdorster E, Oberdorster J (2005) Nanotoxicology: An emerging discipline evolving from studies of ultrafine particles. Environ. Health Perspect. 113: 823-839

PEN. (2010). "An inventory of nanotechnology-based consumer products currently on the market." Retrieved Nov. 11, 2010, from http://www.nanotechproject.org/inventories/consumer/.

Royal Society (2004) Nanoscience and nanotechnologies: Opportunities and uncertainties. Science Policy Section, The Royal Society and the Royal Academy of Engineering. London.

Savolainen K, Alenius H, Norppa H, Pylkkanen L, Tuomi T, Kasper G (2010) Risk assessment of engineered nanomaterials and nanotechnologies--a review. Toxicology 269: 92-104

Schneider T, Brouwer DH, Koponen IK, Jensen KA, Fransman W, Van Duuren-Stuurman B, Van Tongeren M, Tielemans E (2011) Conceptual model for assessment of inhalation exposure to manufactured nanoparticles. J Expo Sci Environ Epidemiol 21: 450-463

Seipenbusch M, Binder A, Kasper G (2008) Temporal evolution of nanoparticle aerosols in workplace exposure. Ann Occ Hyg 52: 707-716

Szymczak W, Menzel N, Keck L (2007) Emissions of ultrafine copper particles by universal motrols controlled by phase angle modulation. J Aerosol Sci 38: 520-531 
US EPA (2007) Nanotechnology white paper. Office of the Science Advisor. Science Policy Council. United State Environmental Protection Agency. Washington, DC: EPA 100/B-07/001

US EPA (2009) Integrated science assessment for particulate matter. National Center for Environmental Assessment - RTP Division; Office of Research and Development; United States Environmental Protection Agency. Research Triangle Park, NC: EPA/600/R-608/139F

van Hemmen JJ, Auffarth J, Evans PG, Rajan-Sithamparanadarajah B, Marquart H, Oppl R (2003) Riskofderm: Risk assessment of occupational dermal exposure to chemicals. An introduction to a series of papers on the development of a toolkit. Ann Occup Hyg 47: 595-598

van Tongeren M. (2011). "Project final report: Development of exposure scenarios for nanomaterials (NANEX). FP7 project number 247794." Retrieved Sept. 7, 2011, from www.nanex-project.eu.

van Veen M (1995) Consexpo, a program to estimate consumer product exposure and uptake. RIVM. Bilthoven, the Netherlands.

von der Kammer F, Legros S, Larsen E, Loescher K, Hofmann T (2011) Separation and characterization of nanoparticles in complex food and environmental samples by field-flow fractionation. Trac-Trends in Analytical Chemistry 30: 425-436

Zartarian V, Bahadori T, McKone T (2005) Adoption of an official ISEA glossary. J Expo Sci Environ Epidemiol. 15: 1-5 